PROCEEDINGS OF THE

AMERICAN MATHEMATICAL SOCIETY

Volume 125, Number 4, April 1997, Pages 943-950

S 0002-9939(97)03956-7

\title{
REDUCED GORENSTEIN CODIMENSION THREE SUBSCHEMES OF PROJECTIVE SPACE
}

\author{
ANTHONY V. GERAMITA AND JUAN C. MIGLIORE
}

(Communicated by Eric M. Friedlander)

\begin{abstract}
It is known, from work of Diesel, which graded Betti numbers are possible for Artinian Gorenstein height three ideals. In this paper we show that any such set of graded Betti numbers in fact occurs for a reduced set of points in $\mathbb{P}^{3}$, a stick figure in $\mathbb{P}^{4}$, or more generally, a good linear configuration in $\mathbb{P}^{n}$. Consequently, any Gorenstein codimension three scheme specializes to such a "nice" configuration, preserving the graded Betti numbers in the process. This is the codimension three Gorenstein analog of a classical result of arithmetically Cohen-Macaulay codimension two schemes.
\end{abstract}

\section{INTRODUCTION}

There are a number of well-known results on arithmetically Cohen-Macaulay (aCM) codimension two subschemes of projective space which fairly recently have been shown to have striking analogs for codimension three arithmetically Gorenstein subschemes. This paper gives a new analog to a classical result.

The starting point for the modern results on codimension three Gorenstein schemes is the well-known structure theorem of D. Buchsbaum and D. Eisenbud [5]. There, the authors showed that there is one matrix which determines the resolution, just as is done by the Hilbert-Burch matrix in codimension two. Then R. Stanley [24] showed that a symmetric sequence of integers is the Hilbert function of an Artinian codimension three Gorenstein algebra if and only if the first difference of the "first half" is the Hilbert function of a codimension two (aCM) Artinian algebra. These two results point the way toward a series of results for Gorenstein codimension three schemes, based on the defining matrix or on the Hilbert function, that are exactly analogous to standard results for the aCM codimension two case. We recall some of these.

G. Ellingsrud [11] proved that the family of codimension two aCM schemes with the same Hilbert function is irreducible. It is also known that the family of codimension two aCM schemes with the same Hilbert function and degrees of generating sets for the homogeneous ideal is irreducible (cf. for instance [4], [7]). Both of these results have been proved for the Gorenstein codimension three case by S. Diesel [10]. Ellingsrud also proved that every aCM codimension two scheme

Received by the editors July 24, 1995.

1991 Mathematics Subject Classification. Primary 14M05, 14C05, 13 D02.

(C)1997 American Mathematical Society 
is non-obstructed; the analogous statement for the Gorenstein codimension three case was proved independently by Miró-Roig [21] and Kleppe [18].

Another important class of results concerns the existence of smooth, or at least integral, aCM schemes in codimension two. A necessary and sufficient condition in terms of the degrees of entries of the Hilbert-Burch matrix is given for curves in $\mathbb{P}^{3}$ by T. Sauer [23] (see also [13]). This was generalized to the codimension two aCM case by G. Bolondi and J. Migliore [3] (see also M. Chang [6]). A precise analog of these results for the integral Gorenstein codimension three case was provided by J. Herzog, N. Trung and G. Valla [17]. From the point of view of the Hilbert function, the necessary and sufficient condition for the existence of smooth aCM curves in $\mathbb{P}^{3}$ (or irreducible codimension two aCM schemes in $\mathbb{P}^{n}$ ) was given by $\mathrm{C}$. Peskine and L. Szpiro [22] and by R. Maggioni and A. Ragusa [19] (see also [13]). This has a precise analog (using Stanley's result above) for integral Gorenstein codimension three schemes, proved by E. De Negri and G. Valla [9].

In the present paper, we give a Gorenstein analog to another classical result. Namely, it was shown by F. Gaeta [12] that every aCM curve in $\mathbb{P}^{3}$ specializes to a stick figure, i.e. a reduced union of lines having only nodes as singularities, and that this can be done even preserving the degrees of the minimal generators of the ideal. (See also [7] and [4] for generalizations to the codimension two aCM case.) In this paper we show that the same is true for Gorenstein codimension three. Stick figures have played an important role in the study of space curves, culminating in the recent negative solution by Hartshorne [16] of the classical Zeuthen Problem, which asks if every smooth curve in $\mathbb{P}^{3}$ specializes to a stick figure. We are not aware of any similar results in higher codimension.

We now give a more precise description of what we do here. Using the structure theorem of Buchsbaum and Eisenbud [5], Diesel described in [10] all the possible graded Betti numbers of minimal free resolutions of Artinian codimension three Gorenstein ideals, leaving open the question of which resolutions (i.e. which graded Betti numbers) can be realized by "nice" subschemes of projective space. In this paper we show that all of the resolutions produced in [10] can be realized by reduced sets of points in $\mathbb{P}^{3}$ (Theorem 2.1). More surprisingly, we show that they can all be realized by stick figures in $\mathbb{P}^{4}$ (Corollary 2.4), and more generally by "good linear configurations" in $\mathbb{P}^{n}$ (Remark 2.5). And furthermore, we show that any Gorenstein codimension three scheme specializes to one of these "nice" Gorenstein schemes with the same resolution.

It is a fairly standard fact in liaison theory [22] that the sum of the ideals of two geometrically linked aCM subschemes of projective space is the saturated ideal of an arithmetically Gorenstein subscheme of codimension one greater. This idea has been used recently by T. Harima [15] to construct specific Artinian Gorenstein ideals achieving all possible Hilbert functions for codimension three. It was further exploited by A. V. Geramita, M. Pucci and Y. S. Shin [14] to find good points in the parametrizing space for Gorenstein codimension three ideals. Both of these papers achieved their results by studying linked sets of points in $\mathbb{P}^{2}$.

In this paper we produce our Gorenstein schemes by studying linked codimension two schemes of higher dimension, taking care to control the objects being linked. It is indicative of the strong connections between the codimension two aCM case and the codimension three Gorenstein case that we use the standard techniques from the former to get our new results about the latter. 


\section{The CONSTRUCTION}

By "any minimal free resolution which occurs" we mean only the graded Betti numbers of the resolution, not the maps themselves. By "Gorenstein" we mean "arithmetically Gorenstein," and by "aCM" we mean "arithmetically CohenMacaulay." For convenience we begin with the case of points in $\mathbb{P}^{3}$.

Theorem 2.1. Any minimal free resolution which occurs for Gorenstein Artinian ideals of height 3 actually occurs for some reduced set of points in $\mathbb{P}^{3}$.

Proof. Let $I$ be a height three Gorenstein ideal in a polynomial ring $S$. Consider a minimal free resolution

$$
0 \rightarrow S(-f) \rightarrow \bigoplus_{i=1}^{2 k+1} S\left(-b_{i}\right) \rightarrow \bigoplus_{i=1}^{2 k+1} S\left(-a_{i}\right) \rightarrow I \rightarrow 0
$$

where $a_{1} \leq \cdots \leq a_{2 k+1}$ and $b_{1} \geq \cdots \geq b_{2 k+1}$. We have from [5] that $b_{i}=f-a_{i}$ for all $i$, and $f+\sum a_{i}=\sum b_{i}$. From this, a quick calculation gives that

$$
\sum_{i=1}^{k+1} a_{i}=\left(f-a_{k+2}\right)+\cdots+\left(f-a_{2 k+1}\right) .
$$

Now let $S=k\left[X_{0}, \ldots, X_{3}\right]$ where $k$ is algebraically closed. We first claim that there exists an aCM curve $C$ in $\mathbb{P}^{3}$ with minimal free resolution

$$
0 \rightarrow \bigoplus_{j=k+2}^{2 k+1} S\left(-f+a_{j}\right) \stackrel{A}{\rightarrow} \bigoplus_{i=1}^{k+1} S\left(-a_{i}\right) \rightarrow I_{C} \rightarrow 0 .
$$

Since we have (1), it is enough to show that the entries on the main diagonal of the degree matrix of $A$ are $>0$ (cf. [13]). That is, we need to show that

$$
\begin{aligned}
\left(f-a_{k+2}\right)-a_{k+1} & >0 \\
\left(f-a_{k+3}\right)-a_{k} & >0 \\
\vdots & \\
\left(f-a_{2 k+1}\right)-a_{2} & >0 .
\end{aligned}
$$

Let $r_{i}=b_{i}-a_{i}=f-2 a_{i}$. We know from [10], Proposition 3.1, that

$$
\begin{aligned}
& r_{i}+r_{2 k+1-i+2}>0 \text { for } i=2, \ldots, \frac{2 k+1+1}{2} \\
& \quad \| \\
& r_{i}+r_{2 k+3-i} .
\end{aligned}
$$

But this means that $f-2 a_{i}+f-2 a_{2 k+3-i}>0$, i.e. $f-a_{i}-a_{2 k+3-i}>0$ for $i=2, \ldots, k+1$, as claimed.

Next we show how to construct a zeroscheme (not necessarily reduced for now) in $\mathbb{P}^{3}$ with the desired resolution. Note that the regularity of $I_{C}$ is $f-a_{k+2}-1<f-a_{1}$ and that (by Gaeta's result, mentioned in the introduction) $C$ can be chosen to be reduced. We will see shortly that we can, in fact, choose $C$ so that we can link it using surfaces $F_{1}$ and $F_{2}$ of degrees $a_{1}$ and $f-a_{1}$ respectively, and get a residual curve $C^{\prime}$ with no component in common with $C$ (and in fact meeting $C$ "nicely"). We accept this for now to do a computation. 
Let $\mathbf{F}$. and $\mathbf{F}_{\bullet}^{\prime}$ be the minimal free resolutions of $I_{C}$ and $I_{C^{\prime}}$ respectively. We know [22] that $I_{C}+I_{C^{\prime}}$ is a codimension three Gorenstein ideal. But from the exact diagram

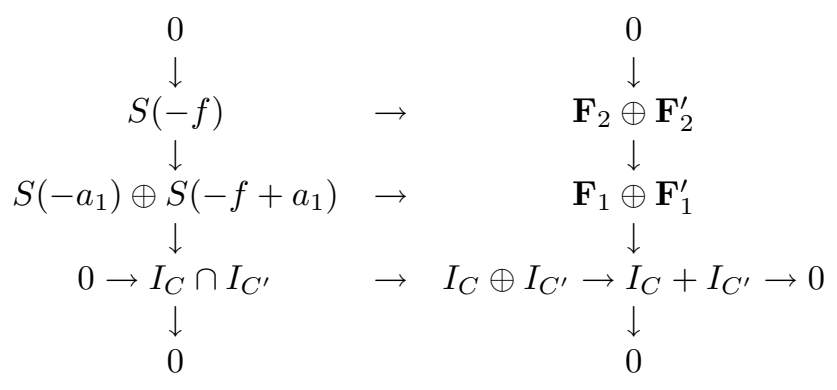

we see, furthermore, that $I_{C}+I_{C^{\prime}}$ is the saturated ideal of a Gorenstein zeroscheme with socle degree $f-3$ (i.e. the last twist is $-f$ ), which is the desired one. To prove that $I_{C}+I_{C^{\prime}}$ has the desired resolution, it is enough to prove that it has the right degrees of generators.

First we note that by the mapping cone [22], and splitting off a redundant $S\left(-f+a_{1}\right)$ (since $F_{1}$ is a minimal generator of $I_{C}$ ), we get that $I_{C^{\prime}}$ has a minimal free resolution $\mathbf{F}^{\prime}$ of the form

$$
0 \rightarrow \bigoplus_{i=2}^{k+1} S\left(-f+a_{i}\right) \rightarrow \bigoplus_{j=k+2}^{2 k+1} S\left(-a_{j}\right) \oplus S\left(-a_{1}\right) \rightarrow I_{C^{\prime}} \rightarrow 0
$$

Hence $I_{C}+I_{C^{\prime}}$ is generated by forms of degrees $a_{1}, \ldots, a_{k+1}$ (from $I_{C}$ ) and $a_{k+2}, \ldots, a_{2 k+1}, a_{1}$ (from $I_{C^{\prime}}$ ). They have in common the form $F_{1}$ of degree $a_{1}$, so we get that $I_{C}+I_{C^{\prime}}$ is generated by forms of the $2 k+1$ desired degrees. We now have to show that it is minimally generated by these forms.

To see why this is so, notice that by the last inequality in (3), $f-a_{2}>a_{2 k+1}$, we also get that $f-a_{1}>a_{2 k+1}$ (since $a_{1}$ is the smallest degree). Suppose that the $2 k+1$ forms do not form a minimal generating set for $I_{C}+I_{C^{\prime}}$. Clearly the form of degree $a_{1}$ can be chosen to be a minimal generator. Let

$$
\begin{gathered}
I_{C}=\left\{F_{1}, G_{2}, \ldots, G_{k+1}\right\}, \\
I_{C^{\prime}}=\left\{F_{1}, G_{k+2}, \ldots, G_{2 k+1}\right\} .
\end{gathered}
$$

Suppose that one of the $G$ 's is a linear combination of the remaining forms. Without loss of generality, say it is one of the minimal generators of $I_{C^{\prime}}$, say $G_{t}$. Then

$$
G_{t}=A_{1} F_{1}+\sum_{i=2}^{k+1} A_{i} G_{i}+\sum_{\substack{j=k+2 \\ j \neq t}}^{2 k+1} B_{j} G_{j}
$$

Hence

$$
G_{t}-\sum_{\substack{j=k+2 \\ j \neq t}}^{2 k+1} B_{j} G_{j}=A_{1} F_{1}+\sum_{i=2}^{k+1} A_{i} G_{i} \in I_{C} \cap I_{C^{\prime}}=\left(F_{1}, F_{2}\right)
$$


But for all $i=k+2, \ldots, 2 k+1, \operatorname{deg} G_{i} \leq a_{2 k+1}<f-a_{2} \leq f-a_{1}=\operatorname{deg} F_{2}$, so

$$
G_{t}-\sum_{\substack{j=k+2 \\ j \neq t}}^{2 k+1} B_{j} G_{j}=B F_{1}
$$

for some $B$, contradicting the fact that $G_{t}$ is a minimal generator of $I_{C^{\prime}}$. This proves that $I_{C}+I_{C^{\prime}}$ is the saturated ideal of a Gorenstein zeroscheme $Z$ in $\mathbb{P}^{3}$ with the desired resolution, as long as we show that such a $C$ and $C^{\prime}$ can be found which are directly linked and which have no components in common.

We now show that not only can this be done, but in fact it can be done in such a way that $Z$ is reduced. The idea is to construct $C$ and the linked $C^{\prime}$ so that the union $X$ is a stick figure, i.e. a reduced union of lines with only nodes as singularities. Then the ideal $I_{C}+I_{C^{\prime}}$ will be the saturated ideal of the scheme-theoretic intersection of $C$ and $C^{\prime}$, and since any component of $C$ meets any component of $C^{\prime}$ at most transversally, this intersection will be reduced.

From the resolution (2), we get that the degree matrix of the presentation matrix $A$ for $I_{C}$ is a $k \times(k+1)$ matrix of the form

$$
\left[\begin{array}{cc}
f-a_{k+2}-a_{k+1} & f-a_{k+2}-a_{k} \cdots \\
f-a_{k+3}-a_{k+1} & f-a_{k+3}-a_{k} \cdots \\
\vdots & \vdots
\end{array}\right]
$$

As we have seen, the entries on the main diagonal (i.e. beginning with the upper left-hand corner) are strictly positive, as hence is the diagonal immediately above it (which ends at the bottom right-hand corner). We extend somewhat an idea of [12] and [7]. Form a matrix of homogeneous polynomials as follows. Let $P_{i, i}$, $1 \leq i \leq k$, be a product of generically chosen linear forms in $S=k\left[X_{0}, \ldots, X_{3}\right]$, where $\operatorname{deg} P_{i, i}=f-a_{k+1+i}-a_{k+2-i}$. Similarly, let $Q_{i, i+1}, 1 \leq i \leq k$, be a product of generically chosen linear forms, where $\operatorname{deg} Q_{i, i+1}=f-a_{i+1+i}-a_{i+1-i}$. Consider the matrix

$$
\left[\begin{array}{ccccccc}
P_{1,1} & Q_{1,2} & 0 & 0 & \ldots & 0 & 0 \\
0 & P_{2,2} & Q_{2,3} & 0 & \ldots & 0 & 0 \\
0 & 0 & P_{3,3} & Q_{3,4} & \ldots & 0 & 0 \\
0 & 0 & 0 & P_{4,4} & \ldots & 0 & 0 \\
\vdots & \vdots & \vdots & \vdots & & \vdots & \vdots \\
0 & 0 & 0 & 0 & \ldots & P_{k, k} & Q_{k, k+1}
\end{array}\right] .
$$

This has the degree matrix given above. Furthermore, since the last degree subminor is $F_{1}=P_{1,1} \cdots P_{k, k}$ and the largest is $G=Q_{1,2} \cdots \cdots Q_{k, k+1}$, and these have no common factor, we see that $A$ is the Hilbert-Burch matrix of an aCM curve $C$ in $\mathbb{P}^{3}$. By the general choice of the $P_{i, i}$ and the $Q_{i, i+1}$, the complete intersection of $F_{1}$ and $G$ is in fact a stick figure (since any three of the planes meet exactly in a point and any four have no common point). As a consequence, the subset $C$ is also a stick figure. But furthermore, $C$ is directly linked by $F_{1}$ and $G$ to a curve (stick figure) $C_{1}$, where $C$ and $C_{1}$ meet in a reduced set of points. But now replace $G$ by $F_{2}$, where $F_{2}$ is the product of $G$ with $f-a_{1}-a_{k+1}$ general linear forms. This gives the desired stick figure link between $C$ and $C^{\prime}$.

Remark 2.2. The construction used in this paper is based on the fact that the sum of the ideals of two geometrically linked aCM schemes is a Gorenstein scheme of 
codimension one greater [22]. We saw that all possible resolutions for Gorenstein codimension three schemes can be obtained using this fact, and they can even be made to be very nice. It is natural to ask whether, in fact, all Gorenstein ideals can be so obtained. In codimension two the answer is yes: a Gorenstein scheme in codimension two is just a complete intersection, so the ideal is the sum of the ideals of the corresponding hypersurfaces. (The condition that the hypersurfaces be geometrically linked is just that they have no common component, which is exactly what we need to get the Gorenstein codimension two scheme.)

In codimension three, we do not yet know the answer. However, notice that in the proof of Theorem 2.1 we were able to obtain all possible resolutions of Gorenstein zeroschemes in $\mathbb{P}^{3}$ using very specific curves. Namely, the aCM curve $C$ we used had generators of degrees taken from the lowest "half" of the generator degrees for our desired resolution, and then $C^{\prime}$ was obtained from $C$ by a specific link. We now show that at least if we restrict to this procedure, it is not possible to obtain all Gorenstein zeroschemes in $\mathbb{P}^{3}$.

We begin with a general set $Z_{1}$ of 8 points in $\mathbb{P}^{2}$. Let $X$ be a complete intersection in $\mathbb{P}^{3}$ of surfaces $F_{1}, F_{2}$ and $F_{3}$ of degrees 3,3 and 4 respectively, which are minimal generators for $I_{Z_{1}}$ (as a subscheme of $\mathbb{P}^{3}$ ), and such that $F_{1}$ and $F_{2}$ form a smooth complete intersection curve. $X$ links $Z_{1}$ to a scheme $Z_{2}$ of degree 28 , which of course lies on the smooth complete intersection curve given by the two cubic surfaces. One can check using the properties of Hilbert functions under liaison [8] that the $h$-vector of the residual set $Z_{2}$ is 1368631 . One can furthermore check that $Z_{2}$ is Gorenstein, with generators of degrees $3,3,4,4,4$, by computing the minimal free resolution of $I_{Z_{1}}$, applying the mapping cone [22], and splitting off three redundant terms from the end of the new resolution (since the link was done with three minimal generators of $I_{Z_{1}}$ ). Since the proof of Theorem 2.1 would require us to find a curve $C$ with minimal generators in degrees $3,3,4$, the fact that the two cubics form a smooth complete intersection means that no such $C$ can exist.

The idea of finding a counterexample by making the first two generators be a smooth complete intersection is due to C. Peterson, who found a larger example than ours using the computer program Macaulay [1] and the methods of [20].

As promised, this shows that curves $C, C^{\prime}$ with the numerical properties required in the proof of Theorem 2.1 do not necessarily exist for a given Gorenstein zeroscheme in $\mathbb{P}^{3}$. However, it is conceivable, a priori, that our set $Z_{2}$ above could be the intersection of two geometrically linked aCM curves $C$ and $C^{\prime}$ with other generating sets. For instance, we could not verify that it is not the intersection scheme of two curves, both of degree 10 and genus 12, linked by a quartic and a quintic surface. (These curves would have generators in degrees $3,4,4$.)

Notice that in higher codimension there are more Gorenstein schemes than simply complete intersections. Furthermore, the fact that the sum of the ideals of two geometrically linked aCM schemes $V, V^{\prime}$ is a Gorenstein ideal of codimension one higher holds even if $V$ and $V^{\prime}$ are linked by a Gorenstein ideal rather than by a complete intersection. Therefore we pose the

Question. Can every Gorenstein scheme $Z$ be obtained as the sum of the ideals of two geometrically linked aCM schemes $V$ and $V^{\prime}$ ? If codim $Z=3$, then "linked" means "linked by a complete intersection", but if codim $Z \geq 4$, then "linked" means "linked by a Gorenstein ideal". 
Remark 2.3. Another tempting conjecture, based on Stanley's result [24] mentioned in the introduction, is that up to the "halfway point" of the Hilbert function, the ideal of the Gorenstein codimension three scheme $Z$ actually agrees with the ideal of some aCM codimension two scheme containing $Z$ (since the Hilbert function does). One checks quickly that most complete intersections already offer a counterexample, but it is conceivable, a priori, that they would be the only counterexamples. But in fact, while this "conjecture" seems to be true often, it is not true in general. A counterexample can be produced using Macaulay [1] and the methods of [20]. For instance, we have produced a Gorenstein ideal of degree 184 with $h$-vector 13610 1520242624201510631 , and generators in degrees 5, 6, 7, 8, 8. The halfway point of the Hilbert function is in degree 7 , and we have checked that the ideal in degree 7 is a codimension three complete intersection of degree $5 \cdot 6 \cdot 7=210$.

Corollary 2.4. Every minimal free resolution which occurs for Gorenstein Artinian ideals of height 3 actually occurs for some stick figure in $\mathbb{P}^{4}$. And every codimension three Gorenstein curve in $\mathbb{P}^{4}$ specializes to a stick figure with the same graded Betti numbers.

Proof. The idea is the same as above. We construct two "good" unions of planes, $V$ and $V^{\prime}$, in $\mathbb{P}^{4}$ which are directly linked, and take our stick figure to be the intersection of $V$ and $V^{\prime}$. We simply observe that with the general choice of the $P_{i, i}$ and the $Q_{i, i+1}$, it is now the case that any three of the hyperplanes meet exactly in a line, any four meet in a point, and any five have no common intersection point. This proves the first statement. The second statement follows from the fact [10] that the scheme Gor $_{D}$, parameterizing Gorenstein codimension three schemes having given degree and relation degrees, is irreducible. (In [10] this was proved only in the Artinian case, but the proof is the same in general.)

Remark 2.5. In [2] the authors defined a good linear configuration in $\mathbb{P}^{n}$ to be a locally Cohen-Macaulay reduced union of linear codimension two subvarieties of $\mathbb{P}^{n}$ such that the intersection of any three has dimension at most $n-4$. Extending this definition in the obvious way to codimension three, we have proved that every codimension three Gorenstein subscheme of $\mathbb{P}^{n}$ specializes to a good linear configuration, and that any resolution that can be obtained in the Artinian height three case can in fact also be obtained in $\mathbb{P}^{n}$ with a good linear configuration.

Note added in proof. For some (negative) results related to the question raised in Remark 2.2, see [25].

\section{REFERENCES}

[1] D. Bayer and M. Stillman, Macaulay, a computer system for computing in Commutative Algebra and Algebraic Geometry.

[2] G. Bolondi and J. Migliore, Configurations of Linear Projective Subvarieties, in "Algebraic Curves and Projective Geometry, Proceedings (Trento, 1988)," Lecture Notes in Mathematics, vol. 1389, Springer-Verlag (1989), 19-31. MR 90i:14053

[3] G. Bolondi and J. Migliore, The Lazarsfeld-Rao property on an arithmetically Gorenstein variety, Man. Math. 78 (1993), 347-368. MR 94a:13008

[4] G. Bolondi and R. Miró-Roig, Deformations of Arithmetically Cohen-Macaulay Subvarities of $\mathbb{P}^{n}$, Man. Math. 64 (1989), no. 2, 205-211. MR 90d:14055

[5] D. Buchsbaum and D. Eisenbud, Algebra Structures for Finite Free Resolutions, and some Structure Theorems for Ideals of Codimension 3, Amer. J. of Math. 99 (1977), 447-485. MR 56:11983 
[6] M. Chang, A filtered Bertini-type theorem, J. Reine Angew. Math. 397 (1989), 214-219. MR 90i: 14054

[7] C. Ciliberto, A. V. Geramita and F. Orecchia, Remarks on a Theorem of Hilbert-Burch, Boll. U.M.I. (7) 2-B (1988), 463-483. MR 90a:13037

[8] E. Davis, A. V. Geramita, F. Orecchia, Gorenstein Algebras and the Cayley-Bacharach Theorem, Proc. AMS 93 (1985), 593-597. MR 86k:14034

[9] E. De Negri and G. Valla, The h-vector of a Gorenstein codimension three domain, Nagoya Math. J. 138 (1995), 113-140. MR 96h:13041

[10] S. Diesel, Irreducibility and Dimension Theorems for Families of Height 3 Gorenstein Algebras, Pacific J. of Math. 172 (1966), 365-397. CMP 96:11

[11] G. Ellingsrud, Sur le schéma de Hilbert des variétés de codimension 2 dans $\mathbb{P}^{e}$ á cône de Cohen-Macaulay, Ann. Sc. Ec. Norm. Sup., t. 8, fasc. 4 (1975), 423-431. MR 52:13831

[12] F. Gaeta, Nuove ricerche sulle curve sghembe algebriche di residuale finito e sui gruppi di punti del piano, Ann. di Mat. Pura et Appl., ser. 4, 31 (1950), 1-64. MR 13:156c

[13] A. V. Geramita and J. Migliore, Hyperplane Sections of a Smooth Curve in $\mathbb{P}^{3}$, Comm. Alg. 17 (1989), 3129-3164. MR 90k:14027

[14] A. V. Geramita, M. Pucci and Y. S. Shin, Smooth points of Gor(T), Queen's Papers in Pure and Appl. Math., vol. 102, Queen's Univ., Kingston, Ontario, 1996, pp. 256-297. CMP 96:10

[15] T. Harima, Some examples of unimodal Gorenstein sequences, J. Pure Appl. Algebra 103 (1995), 313-324. CMP 96:03

[16] R. Hartshorne, Families of Curves in $\mathbb{P}^{3}$ and Zeuthen's Problem, preprint.

[17] J. Herzog, N. V. Trung and G. Valla, On hyperplane sections of reduced irreducible varieties of low codimension, J. Math. Kyoto Univ. 34-1 (1994), 47-72. MR 95d:14048

[18] J. Kleppe, Deformations of graded algebras, Math. Scand. 45 (1979), 205-231. MR 82j:13017

[19] R. Maggioni and A. Ragusa, The Hilbert Function of Generic Plane Sections of Curves in $\mathbb{P}^{3}$, Inv. Math. 91 (1988), 253-258. MR 89g:14027

[20] J. Migliore and C. Peterson, Construction of Codimension Three Gortenstein Subschemes of Projective Space, in preparation.

[21] R. Miró-Roig, Non-obstructedness of Gorenstein subschemes of codimension 3 in $\mathbb{P}^{n}$, Beiträge zur Alg. und Geom. 33 (1992), 131-138. MR 93d:14071

[22] C. Peskie and L. Szpiro, Liaison des variétés algébriques. I, Inv. Math. 26 (1974), 271-302. MR 51:526

[23] T. Sauer, Smoothing Projectively Cohen-Macaulay Space Curves, Math. Ann. 272 (1985), 83-90. MR 87c: 14060

[24] R. Stanley, Hilbert Functions of Graded Algebras, Advances in Math. 28 (1978), 57-83. MR 58:5637

[25] B. Ulrich, Sums of linked ideals, Trans. Amer. Math. Soc. 318 (1990), 1-42. MR 90f:13012

(A. V. Geramita) Department of Mathematics and Statistics, Queen's University, Kingston, Ontario, Canada K7L 3N6

E-mail address: tony@mast.queensu.ca

(A. V. Geramita) Dipartimento di Matematica, Universitá di Genova, Genova, Italia

E-mail address: geramita@dima.unige.it

(J. C. Migliore) Department of Mathematics, University of Notre Dame, Notre Dame, INDIANA 46556

E-mail address: Juan.C.Migliore.1@nd.edu 